\title{
Passivation of cadmium in river sediment using phosphate- solubilizing bacteria and nano-hydroxyapatite loaded biochar
}

\section{(HAP@BC)}

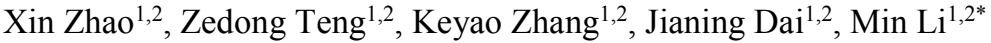 \\ ${ }^{1}$ College of Environmental Science and Engineering, Beijing Forestry University, Beijing 100083, China \\ ${ }^{2}$ Beijing Key Lab for Source Control Technology of Water Pollution. Beijing Forestry University, Beijing \\ 100083, China. (E-mail: minli@,bjfu.edu.cn)
}

\begin{abstract}
Cadmium (Cd) has strong bioaccumulation property and long-lasting toxicity, which is a serious threat to human health, thus it is extremely urgent to remediate the $\mathrm{Cd}$ contaminated river sediments. Nano-hydroxyapatite loaded biochar (HAP@BC) was synthesized and coupled with phosphate solubilizing bacteria (PSB) to enhance the passivation efficiency of cadmium in this research. It has much advantages compared to using HAP@BC only due to the higher dissolution efficiency of phosphorus by PSB. In addition, the passivation efficiency of cadmium via HAP@BC and PSB under different conditions and its mechanism were also investigated. The results showed that the highest $\mathrm{Cd}^{2+}$ removal efficiency could reach $90 \%$ in liquid medium when using PSB cooperated with 5:1 HAP@BC in the condition of temperature $15 \sim 37^{\circ} \mathrm{C}, \mathrm{pH} 7$, initial $\mathrm{Cd}^{2+}$ concentration less than $0.5 \mathrm{mM}$. Furthermore, the simulated sediment experiment indicated that the acid soluble fraction of $\mathrm{Cd}$ was reduced by $28 \%$ and transformed into more stable forms, such as residual fraction. All the experiments of this work demonstrate that the PSB cooperated with HAP@BC is an excellent candidate of passivation agent and could be considered as an effective way for Cd immobilization in contaminated soil in the future.
\end{abstract}

Keyword: River sediment; Cadmium; Immobilization; Phosphate-solubilizing bacteria; Nano-hydroxyapatite loaded biochar. 